\title{
Fibrinolysis Therapy Combined with Deferred PCl versus Primary Angioplasty for STEMI Patients During the COVID-19 Pandemic: Preliminary Results from a Single Center
}

This article was published in the following Dove Press journal:

International Journal of General Medicine

Jing Nan

Shuai Meng

Hongyu $\mathrm{Hu}$

Ruofei Jia

Zening Jin

Department of Cardiology and Macrovascular Disease, Beijing Tiantan Hospital, Capital Medical University,

Beijing, People's Republic of China
Correspondence: Zening Jin Department of Cardiology and Macrovascular Disease, Beijing Tiantan Hospital, Capital Medical University, No. II 9 South Fourth Ring West Road, Fengtai District, Beijing 100070, People's Republic of China

Email Jin_zening@I63.com
Introduction: The efficacy of fibrinolysis therapy with deferred percutaneous coronary angioplasty (FPCI) versus primary angioplasty (PPCI) during the coronavirus disease 2019 (COVID-19) pandemic is unclear when medical quarantine is needed.

Patients and Methods: Acute ST segment elevation myocardial infarction (STEMI) patients underwent PPCI after finishing the screening protocol from January 23, 2020 to June 10, 2020 while FPCI was applied when COVID-19-confirmed cases reoccurred in Beijing near our hospital from June 11, 2020 to July 20, 2020. The door-to-balloon time (DTB) or door-to-needle time (DTN) as well as in-hospital adverse clinical outcomes were compared between the two groups. A propensity score-matched (PSM) analysis was performed to diminish the potential influence of confounding factors on the clinical outcomes. Results: A total of 126 STEMI patients underwent PPCI after finishing the screening protocol and 17 patients received FPCI before PSM. Patients who received FPCI were younger than patients who underwent PPCI (50.8 \pm 14.0 versus $64.1 \pm 14.2$ years, $\mathrm{p}=0.001)$, and chronic kidney disease $(\mathrm{CKD})$ was less common in FPCI patients than in patients who underwent PPCI $(0 \%$ versus $24.6 \%, \mathrm{p}=0.024)$. The DTN was significantly shorter than DTB ( $25.8 \pm 4.2$ versus 61.1 $\pm 10.7, \mathrm{p}=0.000)$ before PSM. The DTN was significantly shorter than DTB $(26.9 \pm 4.2$ versus $64.9 \pm 23.6, \mathrm{p}=0.000$ ); however, the incidence rate of in-hospital ischemia and bleeding adverse clinical outcomes were comparable between the two groups after PSM.

Conclusion: Fibrinolysis therapy combined with deferred PCI can reduce the ischemia time and has a similar in-hospital adverse clinical outcome rate compared with patients who underwent primary PCI during the COVID-19 pandemic.

Keywords: ST elevation myocardial infarction, fibrinolysis therapy, percutaneous coronary intervention, coronavirus disease 2019 , propensity score-matched analysis

\section{Introduction}

The novel coronavirus 2019 (COVID-19) was considered as a global pandemic by World Health Organization (WHO) since March 2020. ${ }^{1}$ Due to its highly contagious features, necessary quarantine and social distance is important in preventing cross-infection. ${ }^{2}$ Health-care facilities, as high-risk regions, applied screening protocols to avoid nosocomial infection. ${ }^{3}$ Not only the patients with chronic conditions are affected, but patients with acute conditions are also compromised during this pandemic. ${ }^{4}$ The negative impact of the COVID-19 pandemic on the management of acute ST segment elevation 
myocardial infarction (STEMI) has been demonstrated by multiple studies with a reduction of catheter activation and increased systemic delay. ${ }^{5,6}$

Current guidelines highlighted the importance of primary percutaneous coronary intervention (PCI) on the management of STEMI because of its superiority of achieving higher rate of TIMI-3 grade flow and lower rate of re-infarction than fibrinolysis therapy. ${ }^{7,8}$ But this recommendation is based on the normal performance of health-care facilities. In fact, fibrinolysis therapy was recommended as the first-line reperfusion therapy (RT) during the COVID-19 pandemic in China, especially in high risk locations. Primary PCI was considered when STEMI patients finished the screening protocol including medical history, blood test and chest computed tomography (CT). ${ }^{9}$ Previous studies have demonstrated that fibrinolysis therapy should be considered when prolonged PCI delay is expected. $^{10-12}$ Which is the better RT for STEMI patients during the COVID-19 pandemic is still unclear.

Beijing Tiantan Hospital, Capital Medical University applied two different RTs according to dynamic change of the cross-infection risk. From January 23, 2020 to June 10, 2020, we performed PPCI in STEMI patients after the patients finished a screening protocol when the overall crossinfection risk was relatively low in Beijing. Fibrinolysis therapy combined with a deferred angioplasty within 24 hours (FPCI) was applied between June 11, 2020 and July 20, 2020 when the second outbreak of COVID-19 began and confirmed COVID-19 patients emerged with possible disease origination from a market nearby our hospital.
We retrospectively collected and analyzed the clinical data of these two groups of patients, and compared the door-to-balloon time (DTB) and door-to-needle time (DTN) as well as the in-hospital adverse clinical outcomes including ischemia and bleeding events.

The study protocol was approved by the Ethics Committee of the Beijing Tiantan Hospital, Capital Medical University. The study was implemented in complete concordance with the tenets of the Declaration of Helsinki on human research. Informed consent was obtained from the study participants.

\section{Patients and Methods Study Design and Participants}

This single center retrospective study was conducted in Beijing Tiantan Hospital, Capital Medical University, Beijing, China. Patients aged 18 years or older diagnosed with acute STEMI within 24 hours after symptom onset in our center from January 23, 2020 to July 20, 2020 were consecutively enrolled. The diagnosis of STEMI was according to the current guidelines, summarized as myocardial ischemia symptoms associated with ST-segment elevation $>2 \mathrm{~mm}$ in $\mathrm{V}_{2}-\mathrm{V}_{3}$ or $>1 \mathrm{~mm}$ in other contiguous leads, or a new (or presumed-to-be new) left bundle branch block. Patients who refused or did not undergo PCI, patients with acute myocardial infarction due to stent thrombosis or those with mechanical complications were excluded. The study flow chart is demonstrated in Figure 1.

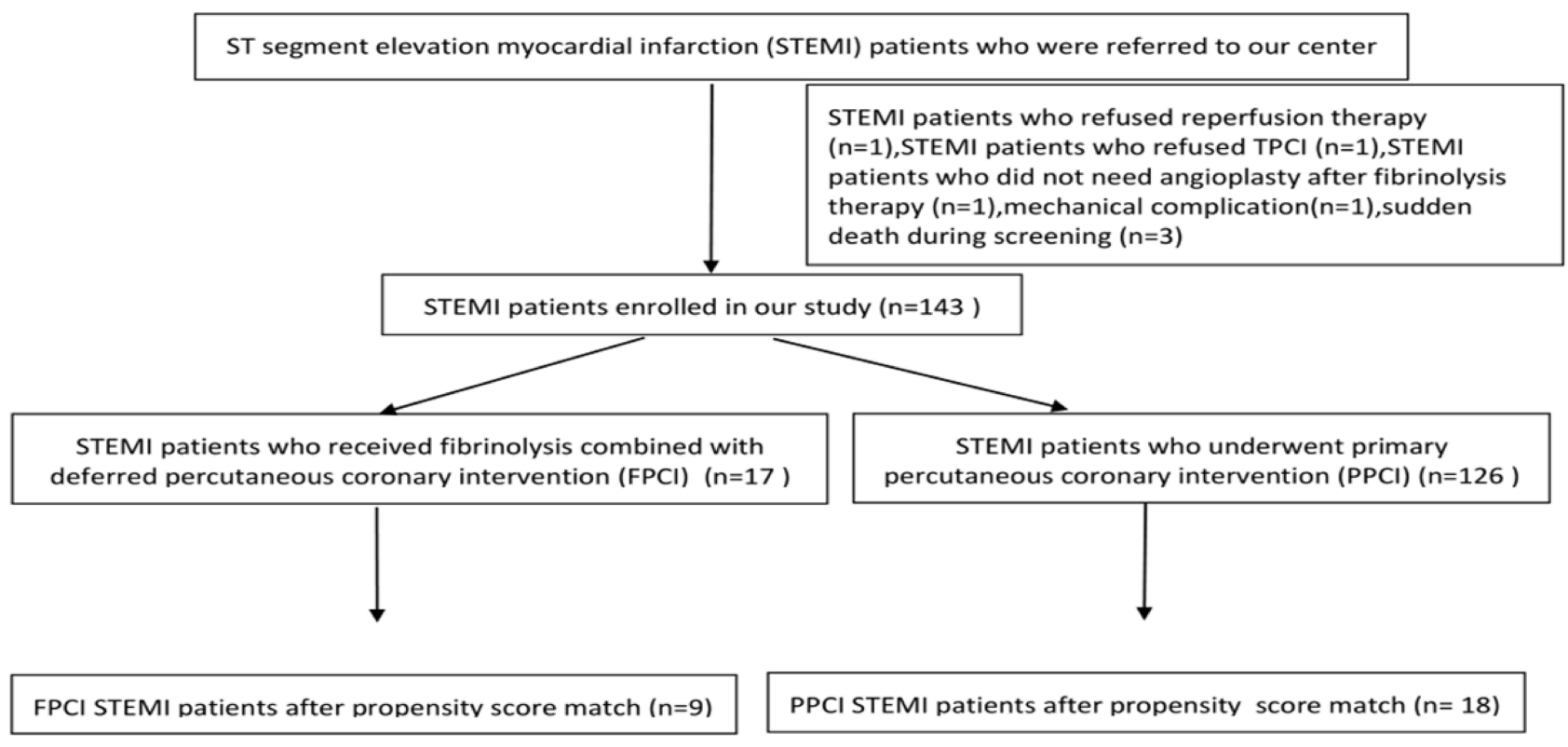

Figure I Study flow chart. 
Clinical information including demographic characteristics, comorbidities and prior medications was collected and analyzed. Clinical manifestation on admission including heart function by Killip classification, on-admission hypotension or cardiac shock was evaluated by professional cardiologists. Risk stratification was evaluated by the Global Registry of Acute Coronary Events (GRACE) and the Thrombolysis in Myocardial Infarction (TIMI) scoring systems, which are widely used to predict the incidence of in-hospital clinical adverse events. ${ }^{13,14}$ The severity of coronary anatomy was scored by Syntax (Synergy between Percutaneous Coronary Intervention with Taxus and Cardiac Surgery) which is a wellaccepted scoring system to evaluate the severity of coronary stenosis. ${ }^{15}$ Laboratory test results which include the peak cardiac troponin I during hospitalization, B-type natriuretic peptide (BNP) on admission, left ventricular ejection fraction (LVEF) on admission were also collected and analyzed.

\section{Screening Protocol Before STEMI Patients Received RT in Beijing Tiantan Hospital During the COVID-19 Pandemic}

STEMI patients who underwent primary PCI had to finish a screening protocol before he or she underwent PPCI between January 31, 2020 and June 10, 2020. If the patient had fever or a suspected medical contact history of Hubei province, then fibrinolysis therapy would be applied. If the patient had no sign of infection and no relevant medical contact history, the patient could undergo primary PCI after finishing the screening tests including whole blood count and chest CT. FPCI was applied from June 11, 2020 to July 20, 2020, when a negative result of nucleic acid as well as blood test and chest CT test results were necessary before performing PCI. Nucleic acid was examined by reversetranscription polymerase chain reaction (RT-PCR) assay. None of the patients who underwent primary PCI were confirmed with COVID-19 infection in our study.

\section{Study Endpoints}

The door-to-needle (DTN) and door to balloon (DTB) times were compared between FPCI patients and PPCI patients. The in-hospital clinical outcomes including ischemia events and bleeding events were also compared. The ischemia events included major adverse cardiac event
(MACE), all-cause of death, cardiac death, non-fatal myocardial infarction, stroke, any revascularization, stent thrombosis, new renal replacement therapy. MACE was defined as the composite of death, myocardial infarction, and revascularization. All deaths were considered cardiac related unless an unequivocal non-cardiac cause could be established. Myocardial infarction was defined by the presence of clinical symptoms associated with electrocardiogram (ECG) changes or abnormal imaging findings indicative of myocardial infarction and an increase in creatine kinase myocardial band fraction above the upper normal limits or an increase in troponin-I above the 99th percentile. Cardiac death was defined as any death due to cardiac or procedure-related causes. Stroke was defined as the presence of a new neurological disorder with signs or symptoms persisting for $24 \mathrm{~h}$ and confirmed by imaging examination. Any revascularization was defined as a repeat PCI or coronary bypass surgery. Stent thrombosis was diagnosed by recommendations from the Academic Research Consortium. ${ }^{16}$ In-hospital adverse events were retrieved from electronic medical records.

\section{Treatment Protocol of $\mathrm{FPCl}$ and PPCl}

Treatment protocol of FPCI: Fibrinolysis therapy was performed in the emergency department using alteplase after excluding contraindications and completion of informed consent. Alteplase was administered $(15 \mathrm{mg}$ intravenous bolus and $0.75 \mathrm{mg} / \mathrm{kg}$ for $30 \mathrm{~min}, 0.5 \mathrm{mg} / \mathrm{kg}$ for $60 \mathrm{~min}$, the total amount is no more than $100 \mathrm{mg}$ ) in combination with low-molecular enoxaparin (30 mg intravenous bolus, subcutaneous injection of $1 \mathrm{mg} / \mathrm{kg}$ after $15 \mathrm{~min}$ and $1 \mathrm{mg} / \mathrm{kg}$ every $12 \mathrm{~h}$ ). A deferred PCI within 24 hours was performed when the negative results of blood test, chest CT as well as nucleic acid test came out. Treatment protocol of PPCI: PPCI was performed after finishing the screening protocol including medical contact history, blood test, chest CT. PCI was done by trained interventionists by standard technique. The access route, application of devices including thrombus aspiration catheter, intra-aortic balloon counter-pulsation (IABP), temporary pacemaker as well as the use of a glycoprotein IIb/IIIa inhibitor (GPI) and anticoagulation medications were all left to the discretion of the operator. All of the patients were prescribed with dual antiplatelet therapy (DAPT) as well as angiotensin-converting enzyme (ACE) inhibitors, angiotensin receptor blocker (ARB), $\beta$ blockers, statins and other medical therapies as the guidelines recommended. 


\section{Statistical Analysis}

Categorical variables were presented as numbers and frequencies (percentages) and were compared using chi-square test or Yate's continuity correction for expected counts per category $<5$. Continuous variables were expressed as mean $\mathrm{SD} \pm$ mean or median (quartile 1 , quartile 3 ) based on normality assumption and were compared using the independent sample $t$-test or Mann-Whitney test, as appropriate. A logistic regression model with propensity scores matching (PSM) of 1:2 ratio was created with variables that were associated with in-hospital clinical outcomes to balance patient characteristics between FPCI and PPCI patients. A $p$ value of $<0.05$ was considered to be significant. Statistical analysis was performed using Statistical Package for the Social Sciences version 22.0 (SPSS Inc., Chicago, IL) and PSM was performed with EmpowerStats 2.20 (X\&Y Solutions Inc., Boston, MA, USA).

\section{Result}

A total of 143 patients were enrolled in this study, of whom 17 patients received FPCI and 126 underwent PPCI before PSM, 9 and 18 patients received FPCI and PPCI after PSM respectively. The process of PSM is shown in Figure 2.

The baseline clinical demographic and characteristics comparison results before and after PSM are shown in Table 1. As shown, FPCI patients were younger than PPCI patients $(50.8 \pm 14.0$ versus $64.1 \pm 14.2$ years, $\mathrm{p}=0.001$ ) before PSM. Also, we observed a significantly lower rate of diagnoses of chronic kidney disease (CKD) in FPCI patients compared with PPCI patients $(0 \%$ versus $24.6 \%, \mathrm{p}=0.024)$ before PSM. There were no significant differences regarding the demographic and clinical characteristics between the two groups after PSM.

\section{Propensity score based on linear model}

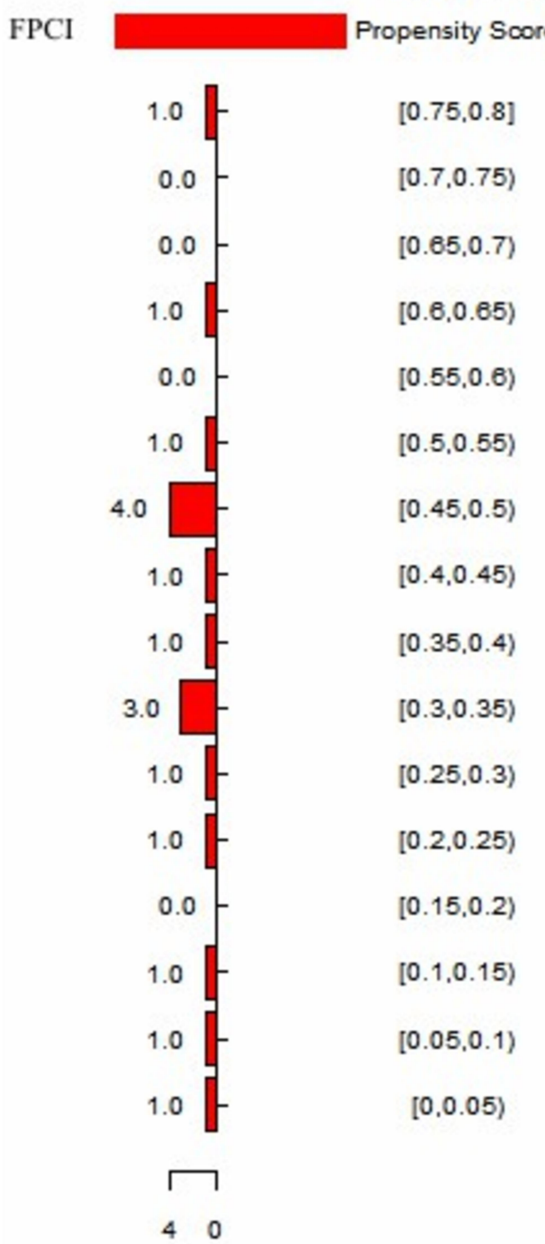

PPCI

21.0

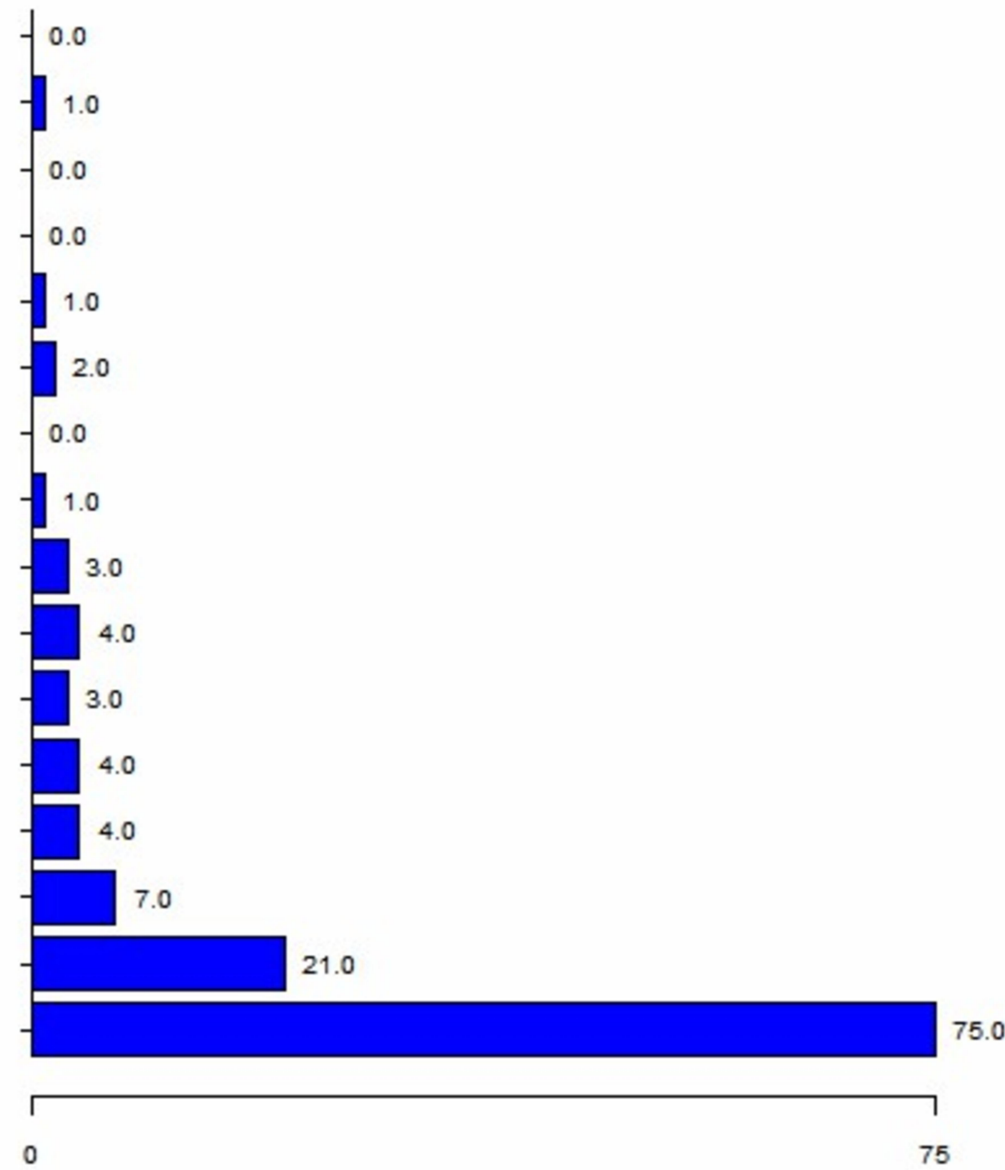

Figure 2 The process of propensity score-matched analysis. 
Table I Comparison of the Baseline Demographic and Clinical Characteristics Between the FPCl and PPCl Patients

\begin{tabular}{|c|c|c|c|c|c|c|}
\hline \multirow[t]{2}{*}{ Variables } & \multicolumn{3}{|l|}{ Before PSM } & \multicolumn{3}{|l|}{ After PSM ${ }^{\#}$} \\
\hline & $\operatorname{FPCI}(n=\mid 7)$ & PPCl $(n=\mid 26)$ & P-value & $\operatorname{FPCl}(n=9)$ & $\operatorname{PPCl}(n=\mid 8)$ & P-value \\
\hline Age/year & $50.8 \pm 14.0$ & $64.1 \pm 14.2$ & 0.001 & $60.67 \pm 10.97$ & $58.00 \pm 11.49$ & 0.569 \\
\hline Male (n,\%) & $14(82.4)$ & $82(65.1)$ & 0.181 & $6(66.7)$ & $15(83.3)$ & 1.000 \\
\hline Smoking (n,\%) & $8(47.1)$ & $44(34.9)$ & 0.421 & $3(33.3)$ & $9(50)$ & 0.681 \\
\hline Hypertension (n,\%) & $9(52.9)$ & $61(48.4)$ & 0.799 & $7(77.8)$ & $8(44.4)$ & 0.218 \\
\hline Diabetes (n,\%) & $6(35.3)$ & $55(43.7)$ & 0.607 & $4(44.4)$ & $4(44.4)$ & 1.000 \\
\hline Dyslipidemia (n,\%) & $10(58.8)$ & $45(35.7)$ & 0.109 & $3(33.3)$ & $10(55.6)$ & 0.496 \\
\hline CKD (n,\%) & $0(0)$ & $3 I(24.6)$ & 0.024 & $0(0)$ & $2(11.1)$ & 0.795 \\
\hline PMI (n,\%) & $3(17.6)$ & $20(15.9)$ & 0.738 & $I(I I . I)$ & $4(22.2)$ & $0.86 \mathrm{I}$ \\
\hline Premature CAD $(n, \%)$ & $5(29.4)$ & $14(|| . \mid)$ & 0.053 & $2(22.2)$ & $\mathrm{I}(5.6)$ & 0.516 \\
\hline $\mathrm{HF}(\mathrm{n}, \%)$ & $2(11.8)$ & $21(16.7)$ & 1.000 & $2(22.2)$ & $5(27.8)$ & 1.000 \\
\hline $\mathrm{AF}(\mathrm{n}, \%)$ & $2(1 I .8)$ & $20(15.9)$ & 1.000 & $\mathrm{I}(\mathrm{II} . \mathrm{I})$ & $2(I I . I)$ & 1.000 \\
\hline Prior PCl (n,\%) & $2(11.8)$ & $14(I \mid .1)$ & 1.000 & $0(0)$ & $I(5.6)$ & 1.000 \\
\hline Prior CABG $(n, \%)$ & $0(0)$ & $2(1.6)$ & 1.000 & $0(0)$ & $0(0)$ & 1.000 \\
\hline ACEI or ARB $(n, \%)$ & $0(0)$ & $21(16.7)$ & 0.077 & $0(0)$ & $2(11.1)$ & 0.795 \\
\hline$\beta$-blockers (n,\%) & & & & I (II.I) & $\mathrm{I}(5.6)$ & 1.000 \\
\hline Statins $(n, \%)$ & $5(29.4)$ & $42(33.3)$ & 1.000 & $2(22.2)$ & $5(27.8)$ & 1.000 \\
\hline
\end{tabular}

Notes: \#The following variables were used in the propensity score (PSM) model: age, sex, history of diabetes, hypertension, dyslipidemia, family history of premature coronary artery disease, smoking, previous myocardial infarction, CKD, AF, HF, previous PCl, previous CABG, previous use of ACEl or ARB, $\beta$-blockers, statins. Bold character indicates that the $P$ value is $<0.05$.

Abbreviations: $\mathrm{FPCl}$, fibrinolysis therapy combined with deferred PCl; PPCl, primary PCl; PSM, propensity score-matched method; CKD, chronic kidney disease; PMI, previous myocardial infarction; CAD, coronary artery disease; $A F$, atrial fibrillation; HF, heart failure; PCl, percutaneous coronary intervention; CABG, coronary artery bypass surgery; ACEI, angiotensin-converting enzyme inhibitor; ARB, angiotensin receptor blocker.

The comparison of angiographic and PCI characteristics between FPCI and PPCI patients before and after PSM is shown in Table 2. Before PSM, both pre- and post-procedure TIMI flow were higher in FPCI patients compared with PPCI patients ( $p=0.000$ and 0.040 , respectively). The GRACE and Syntax risk scores were lower in FPCI patients compared with PPCI patients $(157.8 \pm 9.5$ versus $169.9 \pm 20.3, \mathrm{p}=0.000$ and 24.0 [19.5-26.5] versus 27.5 [22.0-33.0], $\mathrm{p}=0.040$, respectively). Also, the rate of application of thrombus aspiration catheter, vasopressure as well as GPI were lower in FPCI patients compared with PPCI patients $(0 \%$ versus $22.2 \%$, $\mathrm{p}=0.025 ; 23.5 \%$ versus $53.8 \%, \mathrm{p}=0.020 ; 82.4 \%$ versus $96.8 \%$, $\mathrm{p}=0.037$, respectively). There were no significant differences regarding the angiographic and PCI characteristics between FPCI and PPCI patients after PSM except for the higher rate of TIMI-3 flow before procedure in TPIC patients $(\mathrm{p}=0.000)$.

The comparison of DTN in FPCI patients and DTB in PPCI patients as well as the ischemia and bleeding events between these two groups before and after PSM is shown in Table 3. The in-hospital adverse clinical outcome was comparable between the two groups regarding the inhospital ischemia events. The bleeding events score was also comparable between the two groups except for the higher rate of minor bleeding events before PSM.
However there was no significant difference in bleeding events between these two groups after PSM.

\section{Discussion}

In this single-center retrospective observational study, a shorter DTN time is observed in FPCI patients compared with PPCI patients in STEMI patients during the COVID-19 pandemic in our center. Although the incidence rate of minor bleeding event is higher in TPIC patients compared with PPCI patients, a similar rate of in-hospital ischemia and bleeding events is observed between the two groups after adjusting the relative risk factors using PSM method (Figure 3).

The proper RT for STEMI patients is still in debate during the COVID-19 pandemic. How to balance the risk of cross-infection and timely and effective blood flow restoration is an important issue for health-care providers. ${ }^{17}$ Fibrinolysis therapy is believed to have a lower rate of TIMI-2 flow and higher rate of re-infarction compared with PPCI, not to mention the potential bleeding risk caused by fibrinolysis agents. ${ }^{7,8}$ However, this conclusion is based on the normal performance of clinical practice. In fact, fibrinolysis therapy is the preferred RT recommended by the Chinese Cardiovascular Association. ${ }^{9}$ But primary PCI is still the preferred RT in 
Table 2 Comparison of Angiographic and Percutaneous Coronary Intervention Characteristics Between the $\mathrm{FPCl}$ and PPCI Patients

\begin{tabular}{|c|c|c|c|c|c|c|}
\hline \multirow[t]{2}{*}{ Variables } & \multicolumn{3}{|l|}{ Before PSM } & \multicolumn{3}{|l|}{ After PSM $\#$} \\
\hline & $\operatorname{FPCl}(n=\mid 7)$ & $\operatorname{PPCl}(n=\mid 26)$ & P-value & $\operatorname{FPCl}(n=9)$ & PPCI $(n=18)$ & P-value \\
\hline \multicolumn{7}{|l|}{ Infarct-related artery } \\
\hline Left main $(n, \%)$ & $0(0)$ & $\mathrm{I}(0.8)$ & & $0(0)$ & $0(0)$ & \\
\hline $\operatorname{LAD}(\mathrm{n}, \%)$ & II (64.7) & $76(60.3)$ & & $5(55.6)$ & $12(66.7)$ & \\
\hline $\operatorname{LCX}(n, \%)$ & $2(11.8)$ & $13(10.3)$ & & $2(22.2)$ & $3(16.7)$ & \\
\hline $\operatorname{RCA}(n, \%)$ & $4(23.5)$ & $36(28.6)$ & & $2(22.2)$ & $3(16.7)$ & \\
\hline Saphenous vein graft $(n, \%)$ & $0(0)$ & $0(0)$ & 0.757 & $0(0)$ & $0(0)$ & 0.668 \\
\hline \multicolumn{7}{|l|}{ Location of infraction } \\
\hline Anterior $(\mathrm{n}, \%)$ & $12(70.6)$ & $78(61.9)$ & & $6(66.7)$ & $12(66.7)$ & \\
\hline Inferial (n,\%) & $5(29.4)$ & $45(35.7)$ & & $3(33.3)$ & $5(27.8)$ & \\
\hline Lateral $(\mathrm{n}, \%)$ & $0(0)$ & $3(2.4)$ & 0.456 & $0(0)$ & $\mathrm{I}(5.6)$ & 0.940 \\
\hline \multicolumn{7}{|l|}{ Pre-procedural TIMI grade } \\
\hline 0 & $2(11.8)$ & $110(87.3)$ & & $0(0)$ & $15(83.3)$ & \\
\hline 1 & $0(0)$ & $\mathrm{II}(8.7)$ & & $0(0)$ & $3(16.7)$ & \\
\hline 2 & $0(0)$ & $3(2.4)$ & & $0(0)$ & $0(0)$ & \\
\hline 3 & $15(88.2)$ & $2(1.6)$ & 0.000 & $9(100)$ & $0(0)$ & 0.000 \\
\hline \multicolumn{7}{|l|}{ Post-procedural TIMI grade } \\
\hline 0 & $0(0)$ & $0(0)$ & & $0(0)$ & $0(0)$ & \\
\hline 1 & $0(0)$ & $5(4.0)$ & & $0(0)$ & $I(5.6)$ & \\
\hline 2 & $0(0)$ & $21(16.7)$ & & $0(0)$ & $4(22.2)$ & \\
\hline 3 & $17(100)$ & $100(79.4)$ & 0.040 & $9(100)$ & $13(72.2)$ & 0.235 \\
\hline \multicolumn{7}{|l|}{ Risk stratification } \\
\hline TIMI risk score & $5(4-6)$ & $5(5-6)$ & 0.172 & $5.0(4-6)$ & $5.5(5-6)$ & 0.322 \\
\hline GRACE risk score & $157.8 \pm 9.5$ & $169.9 \pm 20.3$ & 0.000 & $155.8 \pm 10.4$ & $166.6 \pm 23.7$ & 0.112 \\
\hline Syntax score & $24.0(19.5-26.5)$ & $27.5(22-33)$ & 0.009 & $22.4 \pm 5.7$ & $27.0 \pm 7.0$ & 0.104 \\
\hline Stent deployment & $17(100)$ & $126(100)$ & 1.000 & $9(100)$ & $18(100)$ & 1.000 \\
\hline \multicolumn{7}{|l|}{ Intra-operation procedure } \\
\hline Thrombus aspiration & $0(0)$ & $28(22.2)$ & 0.025 & $0(0)$ & $3(16.7)$ & 0.529 \\
\hline $\operatorname{IABP}(n, \%)$ & $I(5.9)$ & $34(27.0)$ & 0.072 & $\mathrm{I}(\mathrm{II} . \mathrm{I})$ & $4(22.2)$ & 0.636 \\
\hline Temporary pacemaker $(\mathrm{n}, \%)$ & $0(0)$ & $6(4.8)$ & 1.000 & $0(0)$ & $0(0)$ & 1.000 \\
\hline Vasopressors (n,\%) & $4(23.5)$ & $69(54.8)$ & 0.020 & $3(33.3)$ & $10(55.6)$ & 0.420 \\
\hline Cardiogenic shock (n,\%) & $3(17.6)$ & $17(13.5)$ & 0.708 & $2(22.2)$ & $2(11.1)$ & 0.582 \\
\hline GPI $(n, \%)$ & $14(82.4)$ & $122(96.8)$ & 0.037 & $7(77.8)$ & $18(100)$ & 0.103 \\
\hline
\end{tabular}

Notes: "The following variables were used in the propensity score (PSM) model: age, sex, history of diabetes, hypertension, dyslipidemia, family history of premature coronary artery disease, smoking, previous myocardial infarction, CKD, AF, HF, previous PCI, previous CABG, previously use of ACEI or ARB, $\beta$-blockers, statins. Bold character indicates that the $P$ value is $<0.05$.

Abbreviations: FPCl, fibrinolysis therapy combined with deferred PCl; PPCl, primary PCl; PSM, propensity score-matched method; LAD, left anterior descending artery; LCX, left circumflex artery; RCA, right coronary artery; GRACE, Global Registry of Acute Coronary Events; Syntax, Synergy Between Percutaneous Coronary Intervention With Taxus and Cardiac Surgery; IABP, intra-aortic balloon counter-pulsation; GPI, glycoprotein Ilb/llla inhibitor.

some countries when isolated catheter lab, patient ward as well as personal protective equipment (PPE) is available. $^{18-20}$ Acentralized and specific "Hub" is also demonstrated to be effective to perform PPCI when the systemic delay is not significantly prolonged. ${ }^{21,22}$

Beijing Tiantan Hospital applied different RT for STEMI patients during the COVID-19 pandemic according to the overall risk of cross-infection. When the overall risk of cross-infection was relatively low in our hospital, we applied PPCI after STEMI patents finished the screening protocol. FPCI was applied when the risk of crossinfection was elevated in our hospital.

The first finding of our study is that the DTN time is significantly shorter than D2B time both before and after PSM. Systemic delay is significantly prolonged during the COVDI-19 pandemic. $^{23}$ Daniels et al. believed that the 
Table 3 Comparison of Door-to-Balloon/Needle Time and In-Hospital Adverse Clinical Outcomes Between the FPCI and PPCl Patients

\begin{tabular}{|c|c|c|c|c|c|c|}
\hline \multirow[t]{2}{*}{ Variables } & \multicolumn{3}{|l|}{ Before PSM } & \multicolumn{3}{|l|}{ After PSM ${ }^{\#}$} \\
\hline & $\operatorname{FPCl}(n=17)$ & PPCI $(n=126)$ & P-value & FPCI $(n=9)$ & PPCI $(n=18)$ & P-value \\
\hline DTB/DTN (min) & $25.8 \pm 4.2$ & $6 I .1 \pm 10.7$ & 0.000 & $26.9 \pm 4.2$ & $64.9 \pm 23.6$ & 0.000 \\
\hline All-cause of death $(n, \%)$ & $0(0)$ & $9(7.1)$ & 0.599 & $0(0)$ & $I(5.6)$ & 1.000 \\
\hline Cardiac death $(n, \%)$ & $0(0)$ & $9(7.1)$ & 0.599 & $0(0)$ & $\mathrm{I}(5.6)$ & 1.000 \\
\hline MACE $(n, \%)$ & $0(0)$ & $12(9.5)$ & 0.361 & $0(0)$ & $\mathrm{I}(5.6)$ & 1.000 \\
\hline Non-fatal myocardial infarction $(\mathrm{n}, \%)$ & $0(0)$ & $5(4)$ & 1.000 & $0(0)$ & $0(0)$ & NA \\
\hline Stroke $(n, \%)$ & $0(0)$ & $\mathrm{I}(0.8)$ & 1.000 & $0(0)$ & $0(0)$ & NA \\
\hline Any revascularization $(\mathrm{n}, \%)$ & $0(0)$ & $\mathrm{I}(0.8)$ & 1.000 & $0(0)$ & $0(0)$ & NA \\
\hline Stent thrombosis $(\mathrm{n}, \%)$ & $0(0)$ & $\mathrm{I}(0.8)$ & 1.000 & $0(0)$ & $0(0)$ & NA \\
\hline Major bleeding ${ }^{\S}(n, \%)$ & $0(0)$ & $0(0)$ & NA & $0(0)$ & $0(0)$ & NA \\
\hline Moderate bleeding $g^{\S}(n, \%)$ & I (5.9) & $0(0)$ & 0.119 & $I(I I . I)$ & $0(0)$ & 0.333 \\
\hline Minor bleeding ${ }^{\S}(\mathrm{n}, \%)$ & $5(29.4)$ & $6(4.8)$ & 0.004 & $2(22.2)$ & $3(16.7)$ & 1.000 \\
\hline
\end{tabular}

Notes: \#The following variables were used in the propensity score model: age, sex, history of diabetes, hypertension, dyslipidemia, family history of coronary artery disease, smoking, previous myocardial infarction, chronic kidney disease (CKD), atrial fibrillation (AF), heart failure (HF), previous PCl, previous coronary artery bypass surgery

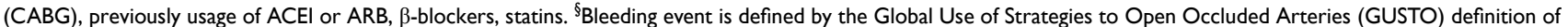
bleeding. Bold character indicates that the $P$ value is $<0.05$.

Abbreviations: $\mathrm{FPCl}$, fibrinolysis therapy combined with deferred $\mathrm{PCl}$; PPCl, primary PCl; PSM, propensity score-matched method; MACE, major adverse cardiac event.

PPCI treatment delays increased during the COVID-19 pandemic, even among COVID-19 negative patients because of the screening protocols. They thought that fibrinolysis therapy might reduce the system delay, which is consistent with our finding. ${ }^{24}$

The second finding of our study is that a higher postprocedural TIMI flow was observed in FPCI patients. It is known that no-reflow or slow re-flow is associated with worse clinical outcomes. $^{25-27}$ The EARLY-MYO Trial (Early Routine Catheterization After Alteplase Fibrinolysis Versus Primary PCI in Acute ST-SegmentElevation Myocardial Infarction) demonstrated that for patients with STEMI presenting $\leq 6$ hours after symptom onset and with an expected PCI-related delay, fibrinolysis therapy with half-dose alteplase and deferred PCI offers more complete myocardial reperfusion when compared with PPCI, which was similar to our finding. ${ }^{28}$

The third finding of our study is that there are no significant differences in the in-hospital ischemia and bleeding events in the two groups after PSM. Multiple studies compared fibrinolysis therapy and PPCI in STEMI patients. Rashid et al. demonstrated that fibrinolysis strategy was associated with similar rates of the composite of mortality, re-infarction, or stroke as compared with PPCI, however, there was a propensity for more bleeding with a fibrinolysis strategy in a realworld study. ${ }^{10}$ Armstrong et al. enrolled STEMI patients who presented within 3 hours after symptom onset and who were unable to undergo primary PCI within 1 hour, these patients were randomly divided into fibrinolysis therapy and primary PCI; it turns out that pre-hospital fibrinolysis with timely coronary angiography resulted in effective reperfusion in patients with early STEMI who could not undergo primary PCI within 1 hour after the first medical contact. However, fibrinolysis was associated with a slightly increased risk of intracranial bleeding. ${ }^{11}$ Strategic Reperfusion Early After Myocardial Infarction (STREAM) trial enrolled patients with STEMI presenting $\leq 3$ hours of symptom onset with an expected time delay from first medical contact (FMC) to PPCI $>1$ hour, and showed no significant difference in the primary cardiovascular events at 30day and 1-year follow-up between PPCI and fibrinolysis patients. ${ }^{12}$ A meta-analysis which enrolled four trials and 1570 patients indicated that deferred PCI was associated with a lower incidence of no-reflow and similar all-cause mortality after 34 months follow up compared with PPCI. ${ }^{29}$ Recently, Danchin et al. reported that $28 \%$ of all patients who underwent late PCI ( $>120 \mathrm{~min}$ from ECG) have poorer 5-year outcomes compared with patients who received fibrinolysis therapy alone. ${ }^{30}$ All of these studies are consistent with our findings. However, the 16 years follow-up result of the Danish Trial in Acute Myocardial Infarction 2 (DANAMI-2) showed that PPCI produced better clinical outcomes compared with fibrinolysis therapy, but this partially 


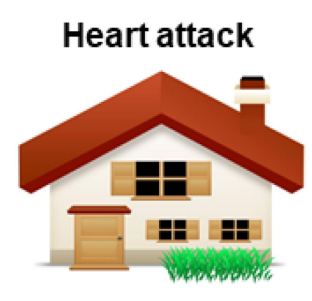

Primary PCl(PPCl)

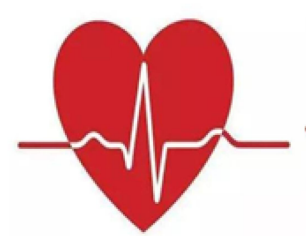

Fibrinolysis therapy with deferred $\mathrm{PCl}$ (FPCl)

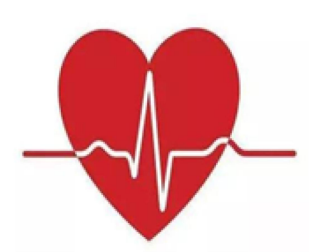

Arrival at hospital

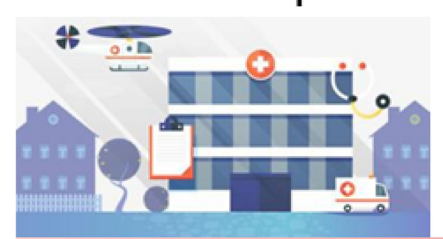

\section{Door-to-balloon time}
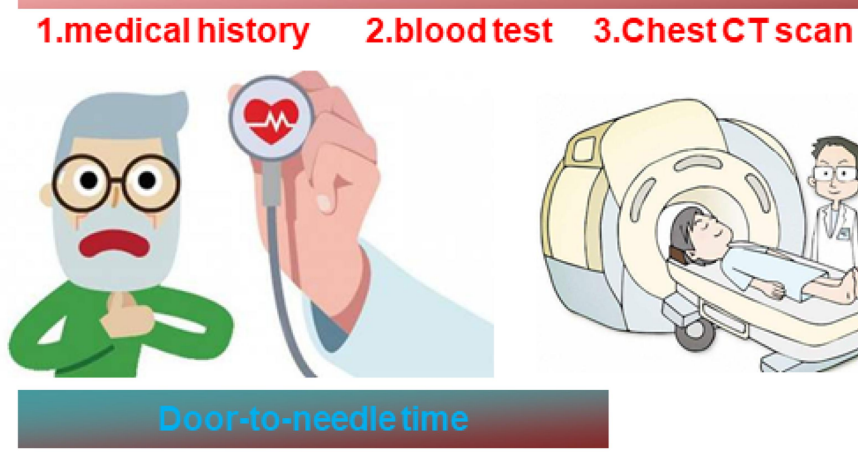

1.medical history 2.blood test 3.Chest CT scan 4.Nucleic acid Deferred PCI
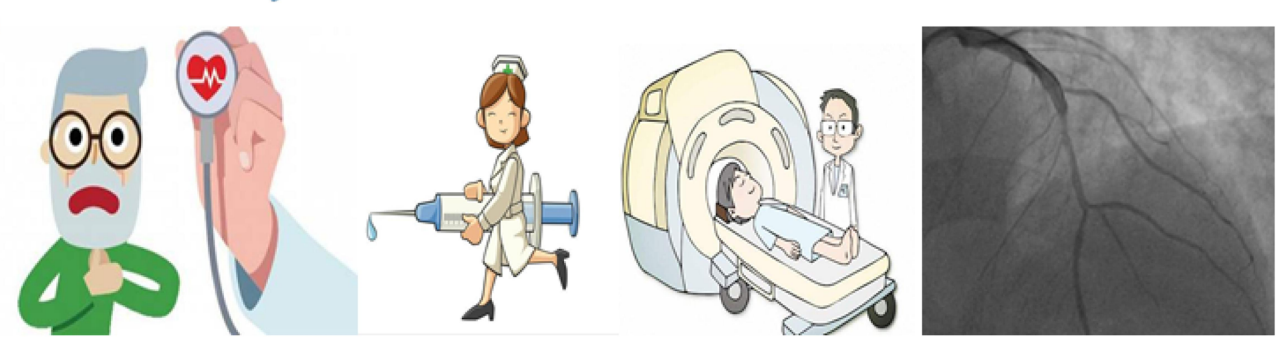

Figure 3 Graphic demonstration of the main results.

was a result of longer delay times compared with other studies. $^{31,32}$

In conclusion, our study for the first time demonstrated that FPCI had better post-procedure TIMI flow and similar incidence rate of in-hospital ischemia and bleeding events after PSM when necessary screening protocol is needed. Due to the limited number of participants and short-time follow-up period, the conclusion still needs to be validated in future studies.

\section{Acknowledgments}

We would like to thank all our coworkers for their brave contributions during the COVID-19 pandemic.

\section{Funding}

This study was funded by the Chinese Cardiovascular Association V.G. Foundation (2017-CCA-VG-042) and Beijing Key Clinical Department Project.

\section{Disclosure}

The authors report no conflicts of interest for this work.

\section{References}

1. Cucinotta D, Vanelli M. WHO declares COVID-19 a Pandemic. Acta Biomed. 2020;91(1):157-160. doi:10.23750/abm.v91i1.9397

2. Abdi S, Salarifar M, Mortazavi SH, et al. COVID-19 sends STEMI to quarantine!? Clin Res Cardiol. 2020;1-2.

3. De Rosa S, Spaccarotella C, Basso C, et al. Reduction of hospitalizations for myocardial infarction in Italy in the COVID-19 era. Eur Heart J. 2020;41(22):2083-2088. doi:10.1093/eurheartj/ehaa409

4. Masroor S. Collateral damage of COVID-19 pandemic: delayed medical care. J Card Surg. 2020;35(6):1345-1347. doi:10.1111/jocs.14638

5. Garcia S, Albaghdadi MS, Meraj PM, et al. Reduction in ST-segment elevation cardiac catheterization laboratory activations in the United States during COVID-19 pandemic. J Am Coll Cardiol. 2020;75(22): S0735-1097. doi:10.1016/j.jacc.2020.04.011

6. Tam CF, Cheung KS, Lam S, et al. Impact of coronavirus disease 2019 (COVID-19) outbreak on outcome of myocardial infarction in Hong Kong, China. Catheter Cardiovasc Interv. 2020. doi:10.1002/ccd.28943

7. Ibanez B, James S, Agewall S, et al. ESC guidelines for the management of acute myocardial infarction in patients presenting with ST-segment elevation: the task force for the management of acute myocardial infarction in patients presenting with ST-segment elevation of the European Society of Cardiology (ESC). Eur Heart J. 2017;2018(39):119-77.

8. Levine GN, Bates ER, Blankenship JC, et al. 2015 ACC/AHA/SCAI focused update on primary percutaneous coronary intervention for patients with ST-Elevation myocardial infarction: an update of the 2011 ACCF/AHA/SCAI guideline for percutaneous coronary intervention and the 2013 ACCF/AHA guideline for the management of ST-Elevation myocardial infarction. J Am Coll Cardiol. 2016;67(10):1235-1250. doi:10.1016/j.jacc.2015.10.005 
9. Han Y, Zeng H, Jiang H, et al. CSC expert consensus on principles of clinical management of patients with severe emergent cardiovascular diseases during the COVID-19 epidemic. Circulation. 2020;141(20): e810-6. doi:10.1161/CIRCULATIONAHA.120.047011

10. Rashid MK, Guron N, Bernick J, et al. Safety and efficacy of a pharmacoinvasive strategy in st-segment elevation myocardial infarction: a patient population study comparing a pharmacoinvasive strategy with a primary percutaneous coronary intervention strategy within a regional system. JACC Cardiovasc Interv. 2016;9(19):2014-2020. doi:10.1016/j.jcin.2016.07.004

11. Armstrong PW, Gershlick AH, Goldstein P, et al. Fibrinolysis or primary PCI in ST-segment elevation myocardial infarction. $N$ Engl $J$ Med. 2013;368(15):1379-1387. doi:10.1056/NEJMoa1301092

12. Sinnaeve PR, Armstrong PW, Gershlick AH, et al. ST-segmentelevation myocardial infarction patients randomized to a pharmaco-invasive strategy or primary percutaneous coronary intervention: strategic reperfusion early after myocardial infarction (STREAM) 1-year mortality follow-up. Circulation. 2014;130 (14):1139-1145. doi:10.1161/CIRCULATIONAHA.114.009570

13. Shuvy M, Beeri G, Klein E, et al. Accuracy of the Global Registry of Acute Coronary Events (GRACE) risk score in contemporary treatment of patients with acute coronary syndrome. Can J Cardiol. 2018;34(12):1613-1617. doi:10.1016/j.cjca.2018.09.015

14. Williams BA, Chagin KM, Bash LD, et al. External validation of the TIMI risk score for secondary cardiovascular events among patients with recent myocardial infarction. Atherosclerosis. 2018;272:80-86. doi:10.1016/j.atherosclerosis.2018.03.026

15. Bundhun PK, Sookharee Y, Bholee A, Huang F. Application of the SYNTAX score in interventional cardiology: a systematic review and meta-analysis. Medicine (Baltimore). 2017;96(28):e7410. doi:10.1097/MD.0000000000007410

16. Cutlip DE, Windecker S, Mehran R, et al. Clinical end points in coronary stent trials: a case for standardized definitions. Circulation. 2007;115 (17):2344-2351. doi:10.1161/CIRCULATIONAHA.106.685313

17. Zeng J, Huang J, Pan L. How to balance acute myocardial infarction and COVID-19: the protocols from Sichuan Provincial People's Hospital. Intensive Care Med. 2020;46(6):1111-1113. doi:10.1007/ s00134-020-05993-9

18. Kirtane AJ, Bangalore S. Why fibrinolytic therapy for ST-segmentelevation myocardial infarction in the COVID-19 pandemic is not your new best friend. Circ Cardiovasc Qual Outcomes. 2020;13(6): e006885. doi:10.1161/CIRCOUTCOMES.120.006885

19. Mahmud E, Dauerman HL, Welt FGP, et al. Management of acute myocardial infarction during the COVID-19 pandemic: a consensus statement from the Society for Cardiovascular Angiography and Interventions (SCAI), the American College of Cardiology (ACC), and the American College of Emergency Physicians (ACEP). Catheter Cardiovasc Interv. 2020. doi:10.1002/ccd.28946

20. Roffi M, Guagliumi G, Ibanez B. The obstacle course of reperfusion for STEMI in the COVID-19 pandemics. Circulation. 2020;141 (24):1951-1953. doi:10.1161/CIRCULATIONAHA.120.047523

21. Ferlini M, Andreassi A, Carugo S, et al. Centralization of the ST elevation myocardial infarction care network in the Lombardy region during the COVID-19 outbreak. Int J Cardiol. 2020;312:24-26. doi:10.1016/j.ijcard.2020.04.062

International Journal of General Medicine

\section{Publish your work in this journal}

The International Journal of General Medicine is an international, peer-reviewed open-access journal that focuses on general and internal medicine, pathogenesis, epidemiology, diagnosis, monitoring and treatment protocols. The journal is characterized by the rapid reporting of reviews, original research and clinical studies

Submit your manuscript here: https://www.dovepress.com/international-journal-of-general-medicine-journal
22. Tumminello G, Barbieri L, Lucreziotti S, et al. Impact of COVID-19 on STEMI: second youth for fibrinolysis or time to centralized approach? Int J Cardiol Heart Vasc. 2020;30:100600. doi:10.1016/j. ijcha.2020.100600

23. Tam CF, Cheung KS, Lam S, et al. Impact of coronavirus disease 2019 (COVID-19) outbreak on ST-segment-elevation myocardial infarction care in Hong Kong, China. Circ Cardiovasc Qual Outcomes. 2020;13(4): e006631. doi:10.1161/CIRCOUTCOMES.120.006631

24. Daniels MJ, Cohen MG, Bavry AA, Kumbhani DJ. Reperfusion of ST-segment-elevation myocardial infarction in the COVID-19 era: business as usual? Circulation. 2020;141(24):1948-1950. doi:10.1161/CIRCULATIONAHA.120.047122

25. Tasar O, Karabay AK, Oduncu V, Kirma C. Predictors and outcomes of no-reflow phenomenon in patients with acute ST-segment elevation myocardial infarction undergoing primary percutaneous coronary intervention. Coron Artery Dis. 2019;30(4):270-276. doi:10.1097/ MCA.0000000000000726

26. Gupta S, Gupta MM. No reflow phenomenon in percutaneous coronary interventions in ST-segment elevation myocardial infarction. Indian Heart J. 2016;68(4):539-551. doi:10.1016/j.ihj.2016.04.006

27. Harrison RW, Aggarwal A, Ou FS, et al. Incidence and outcomes of no-reflow phenomenon during percutaneous coronary intervention among patients with acute myocardial infarction. Am J Cardiol. 2013;111(2):178-184. doi:10.1016/j.amjcard.2012.09.015

28. Pu J, Ding S, Ge H, et al. Efficacy and safety of a pharmaco-invasive strategy with half-dose alteplase versus primary angioplasty in st-segmentelevation myocardial infarction: EARLY-MYO trial (early routine catheterization after alteplase fibrinolysis versus primary PCI in acute ST-segment-elevation myocardial infarction). Circulation. 2017;136 (16):1462-1473. doi:10.1161/CIRCULATIONAHA.117.030582

29. Mahmoud AN, Saad M, Elgendy AY, Mentias A, Elgendy IY. Deferred or immediate stent implantation for primary percutaneous coronary intervention: a meta-analysis of randomized trials. Catheter Cardiovasc Interv. 2018;91(2):260-264. doi:10.1002/ccd.27240

30. Danchin N, Popovic B, Puymirat E, et al. Five-year outcomes following timely primary percutaneous intervention, late primary percutaneous intervention, or a pharmaco-invasive strategy in ST-segment elevation myocardial infarction: the FAST-MI programme. Eur Heart J. 2020;41(7):858-866. doi:10.1093/eurheartj/ehz665

31. Thrane PG, Kristensen SD, Olesen KKW, et al. 16-year follow-up of the Danish Acute Myocardial Infarction 2 (DANAMI-2) trial: primary percutaneous coronary intervention vs. fibrinolysis in ST-segment elevation myocardial infarction. Eur Heart J. 2020;41 (7):847-854. doi:10.1093/eurheartj/ehz595

32. Nielsen PH, Terkelsen CJ, Nielsen TT, et al. System delay and timing of intervention in acute myocardial infarction (from the Danish Acute Myocardial Infarction-2 [DANAMI-2] trial). Am J Cardiol. 2011;108 (6):776-781. doi:10.1016/j.amjcard.2011.05.007

across all disease areas. The manuscript management system is completely online and includes a very quick and fair peer-review system, which is all easy to use. Visit http://www.dovepress.com/ testimonials.php to read real quotes from published authors. 